(1)

CrossMark

\title{
Tuberculosis research questions identified through the WHO policy guideline development process
}

\author{
Nebiat Gebreselassie, Dennis Falzon, Matteo Zignol and Tereza Kasaeva \\ Affiliation: Global TB Programme, World Health Organization, Geneva, Switzerland.
}

Correspondence: Nebiat Gebreselassie, World Health Organization, Global TB Programme, Avenue Appia 20, Geneva 121, Switzerland. E-mail: gebreselassiendawho.int

@ERSpublications

WHO guideline development groups identify research questions using systematic reviews, economic analyses and stakeholder consultations during policy guidance development to identify urgent research gaps in the policy/implementation interface http://ow.ly/lUUw30nQZRO

Cite this article as: Gebreselassie N, Falzon D, Zignol M, et al. Tuberculosis research questions identified through the WHO policy guideline development process. Eur Respir J 2019; 53: 1802407 [https://doi.org/ 10.1183/13993003.02407-2018].

High-quality research evidence is critical for improving global health and health equity, and for achieving the World Health Organization (WHO)'s objective of the attainment of the highest possible level of health by all peoples [1]. This need is most apparent when responding to complex epidemics such as tuberculosis (TB). TB is the leading killer among diseases caused by an infectious agent worldwide, the leading killer of people with HIV infection and a leading cause of death from airborne anti-microbial resistant infections, taking heavy tolls on human lives, communities and health systems at large $[2,3]$. WHO estimates that TB caused illness in 10 million people and claimed an estimated 1.6 million lives in 2017 alone [2]. The WHO End TB Strategy, in the context of the Sustainable Development Goals (SDGs), lays ambitious goals and milestones to end the epidemic by reducing incidence and mortality by $80 \%$ and $90 \%$ in 2030 compared to 2015: such reductions can only be achieved if there are major technological breakthroughs by 2025 [4].

Critical research is needed to acquire rapid point-of-care TB diagnostics, including for drug resistance; shorter, safer and simpler regimens effective against drug-susceptible and drug-resistant $\mathrm{TB}$, as well as latent TB infection (LTBI) that are appropriate for treatment of TB/HIV co-infection; and a new TB vaccine that is effective both before and after exposure. These require scientific advances in the discovery and development of new biomedical tools, together with innovative delivery mechanisms to effectively adapt and adopt new technologies and optimise the necessary linkages and integrations with other health services and sectors. For this reason, "Intensified research and innovation" has been identified as one of the three essential pillars of the End-TB Strategy. This editorial summarises the research questions identified through recent WHO TB policy guidance to increase the quality of evidence for policy-making. Based on evidence arising from research, WHO is mandated to produce recommendations to guide clinical practice and public health policy for TB prevention and care in response to demand from public health decision makers. WHO guideline development groups (GDGs), which include researchers, the health workforce, civil society, as well as end-users of the guidelines, such as policymakers from government, professional associations and other constituencies, are appointed by WHO to develop policy guidelines [5]. A GDG meets with the primary objective of agreeing on the scope of recommendations by reviewing evidence, structured according to the standard framework of population, intervention, control, outcomes (PICO). This permits a systematic study 
of relevant evidence, the formulation of recommendations and the identification of knowledge gaps that need to be addressed through high quality research conducted in various epidemiological, demographic and geographic settings. The research questions highlighted in this document arose because the respective GDGs agreed they were critical for increasing the certainty/strength of existing recommendation, and/or for stimulating the development or optimisation of new recommendations that can lead to improvement in patient health and welfare. This step is an integral part of the WHO guideline development process (see, for example, the discussion section of FALzON et al. [6]).

Among the major challenges facing global policy guidance development in TB are the shortage of good quality evidence exacerbated, for example, by lack of sufficient clinical trials with direct evidence of clinical benefit or improvement in an established surrogate for clinical benefit; data inaccessibility including for programmatic experiences of benefits and safety of interventions in real world setting; or when the evidence being presented does not address broader questions of values and priorities that go beyond medical interventions (e.g. acceptability, feasibility, resource distribution and health equity). Evidence obtained from well-designed, large scale multidisciplinary studies with robust testing of interventions are therefore needed to improve the strength of future guidance.

The most up-to-date WHO policy guidance documents for TB prevention and care are summarised in a Compendium of TB Guidelines and Associated Standards [7, 8]. Using this compendium as a reference, we compiled a list of 155 research questions across the continuum of TB prevention, diagnosis, treatment and care (also summarised in table 1): three related to early detection; 35 related to diagnosis of $\mathrm{TB}$ disease, 10 related to the diagnosis and management of latent TB infection, 38 related to treatment of TB disease, including drug-resistant TB; 38 related to the management of TB/HIV and malnutrition; and 31 related to childhood TB management [10]. Because these research questions are limited in scope to needs identified during guideline development processes, the majority of the questions highlight gaps at the policy/implementation interface (figure 1). Systematically linking such research questions to public health goals requires collaboration among funders, researchers and end users to ensure that funded research

TABLE 1 Research questions from the World Health Organization (WHO) tuberculosis (TB) policy guidance documents

Policy guidance

Research

questions

\section{Early detection of TB}

1.1 Systematic screening for active tuberculosis: principles and recommendations

2. Diagnosing TB disease

2.1 Xpert MTB/RIF and Ultra assays for the diagnosis of pulmonary and extrapulmonary TB in adults and children and WHO Meeting Report of a Technical Expert Consultation: non-inferiority analysis of Xpert MTB/RIF Ultra compared to Xpert MTB/RIF

2.2 The use of loop-mediated isothermal amplification (TB-LAMP) for the diagnosis of pulmonary tuberculosis

2.3 The use of lateral flow urine lipoarabinomannan assay (LF-LAM) for the diagnosis and screening of active tuberculosis in people living with HIV: policy update

2.4 The use of molecular line probe assays for the detection of resistance to isoniazid and rifampicin

2.5 Molecular line probe assays for the detection of resistance to second-line anti-tuberculosis drugs: policy guidance

3. Diagnosing and treating latent TB infection

\section{Treating TB}

4.1 Treatment of drug-susceptible tuberculosis and patient care: 2017 update

4.2 WHO treatment guidelines for isoniazid-resistant TB

4.3 WHO treatment guidelines for drug-resistant tuberculosis: 2016 update

5. TB/HIV and other comorbidities

5.1 Consolidated guidelines on the use of antiretroviral drugs for treating and preventing HIV infection (this is an HIV guideline and the two research questions were those relevant to TB)

5.2 Integrating collaborative TB and HIV services within a comprehensive package of care for people who inject drugs: consolidated guidelines

5.3 WHO policy on collaborative TB/HIV activities

5.4 Guidelines for managing advanced HIV disease and rapid initiation of antiretroviral therapy (this is an HIV guideline and the 4 research questions were those relevant to TB)

5.5 Nutritional care and support for patients with tuberculosis

6. Managing TB in children

6.1 Guidance for national tuberculosis programmes on the management of tuberculosis in children

All guidelines are accessible through the WHO Compendium of TB Guidelines and Associated Standards [7], also available as a mobile phone app. 
represents value for money, not only through the generation of new knowledge but also by contributing to health and economic outcomes. There are several ways of accomplishing that. The National Institute for Health Research Public Health Research Programme (NIHR PHR Programme) in the UK, for example, includes public health decision makers in its decision-making committee, and subsequently, the research it funds has been shown to align with priorities highlighted in national guidelines [11]. However, this is not the practice across all research funders. An exploratory qualitative study of funding strategy among five high-profile public health research funding organisations showed limited involvement from end users/ policymakers in the prioritisation of research questions for funding [12]. Considering the need for well-funded, timely and high quality research for policy, funders should capitalise on opportunities to strengthen participation of policymakers and other end users in generating priority-driven research funding streams.

At a time when there are many competing demands on limited resources, the WHO and its partners, countries, civil society and affected communities have a joint responsibility to ensure that $\mathrm{TB}$ research investments help achieve the goals and targets of the End TB Strategy and the SDGs. In recognition of this need, a TB resolution adopted at the World Health Assembly in May 2018 requested WHO to develop a global strategy for TB research and innovation, "to make further progress in enhancing cooperation and coordination in respect of tuberculosis research and development" [13]. Considering the significant funding gap for TB research (USD 1.3 billion gap in 2017 when benchmarked against the targets outlined in the Global Plan to End TB 2016-2020: the Paradigm Shift), such coordination and collaboration is envisioned to help direct time and resources to the most urgent evidence needs faced by TB policymakers [14-16].

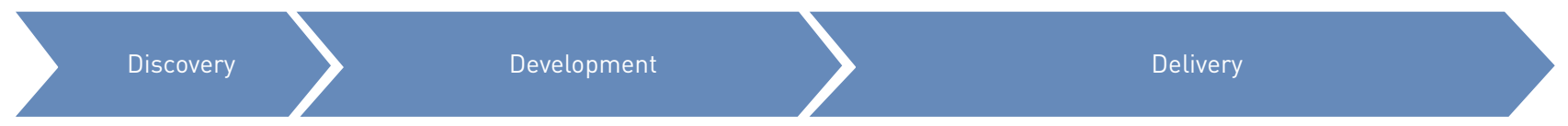

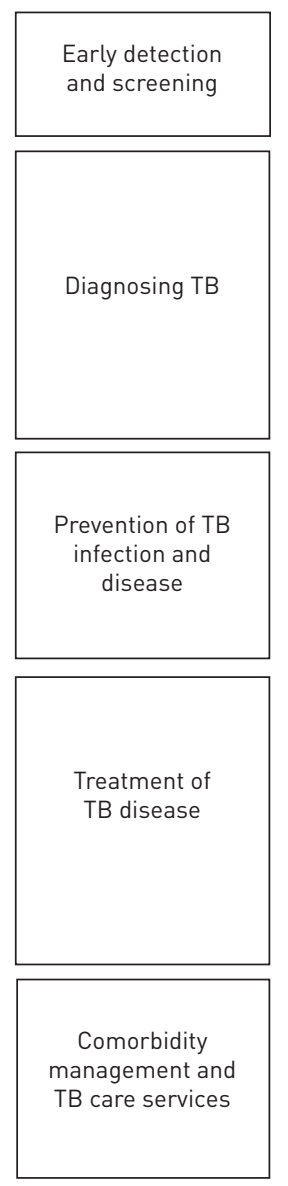

\section{- Develop low cost, noninvasive, point-of-care diagnostics for all forms of TB, effective also for children and people living with HIV}

- Develop better-tolerated and shorter preventive treatment regimens, including for children and people living with HIV

- Develop vaccines that would provide greater protection than BCG

- Develop better-tolerated, more effective and shorter regimens, for treating drug-resistant forms of TB, including in children and people living with HIV

- Investigate the specificity and sensitivity of TB screening strategies, as well as the feasibility, effectiveness and cost-effectiveness of screening for broader indications of TB symptoms

- Investigate the accuracy and detection limit of new diagnostics in different population groups and their impact on the cascade of care and patient outcome - Comparative evaluation of diagnostic algorithms in different epidemiological and geographic settings to assess acceptability, feasibility, affordability and effectiveness

- Assess training competency need for scale up and impact

- Conduct country-specific cost-effectiveness and cost-benefit analyses of new diagnostics

- Identify strategies to improve adherence and treatment completion of patients on TB infection therapy or disease treatment

- Map the epidemiological burden of LTBI and risk of TB transmission in different subpopulations and settings

- Conduct randomised controlled trials with adequate power to guide policy guidance on optimisation of regimens (safety, tolerability and effectiveness) for preventing TB disease (including among MDR-TB contacts), and for treating $\mathrm{Hr}-\mathrm{TB}$

- Assess effective introduction of new regimens under operational settings

- Assess adverse events and pharmacokinetics of patients under preventive and MDR-TB treatment

- Conduct dosage optimisation for extrapulmonary TB treatment

- Conduct cost-effectiveness and cost-benefit analyses of difference service delivery models for MDR-TB treatment

- Conduct country-specific cost-effectiveness and cost-benefit analyses of new treatment regimens

- How to best provide high quality integrated TB/HIV and childhood TB management in a manner that is affordable, accessible, scalable and safe for patients

- Understanding the impact of macronutrient intake/food supplementation on TB treatment outcomes

FIGURE 1 Representation of the research questions documented in World Health Organization tuberculosis (TB) policy guidance documents. BCG: bacille Calmette-Guerin; LTBI: latent TB infection; MDR-TB: multidrug-resistant TB; Hr-TB: isoniazid-resistant TB. 
Conflict of interest: None declared.

\section{References}

Constitution of the World-Health-Organization. Public Health Rep 1946; 61: 1268-1277.

World Health Organization. Global TB Report. Geneva, World Health Organization, 2018.

The Review on Antimicrobial Resistance. Tackling Drug-resistant Infections Globally: Final Report and Recommendations. London, The Review on Antimicrobial Resistance, 2016.

4 Uplekar M, Weil D, Lonnroth K, et al. WHO's new end TB strategy. Lancet 2015; 385: 1799-1801.

5 World Health Organization. Handbook for Guideline Development. 2nd Edn. Geneva, World Health Organization, 2014; p. 179.

6 Falzon D, Schunemann HJ, Harausz E, et al. World Health Organization treatment guidelines for drug-resistant tuberculosis, 2016 update. Eur Respir J 2017; 49: 1602308.

7 World Health Organization. Compendium of TB Guidelines and Associated Standards. Geneva, World Health Organization, 2018.

8 Gilpin C, Korobitsyn A, Migliori GB, et al. The World Health Organization standards for tuberculosis care and management. Eur Respir J 2018; 51: 1800098.

9 World Health Organization, TB/HIV Working Group/Stop TB Partnership. Priority Research Questions for TB/HIV in HIV-prevalent and Resource-limited Settings. Geneva, World Health Organization, 2010.

10 World Health Organization. Evidence Gaps and Research Needs Identified during Tuberculosis Policy Guideline Development. Geneva, World Health Organization, 2018.

11 Martin BR. The Research Excellence Framework and the "impact agenda": are we creating a Frankenstein monster? Res Eval 2011; 20: 247-254.

12 Cartier Y, Creatore MI, Hoffman SJ, et al. Priority-setting in public health research funding organisations: an exploratory qualitative study among five high-profile funders. Health Res Policy Syst 2018; 16: 53.

13 World Health Organization. Preparation for a High-level Meeting of the General Assembly on Ending Tuberculosis (WHA71.3). Geneva, Seventy-first World Health Assembly. World Health Organization, 2018.

14 World Health Organization. Global Investments in Tuberculosis Research and Development: Past, Present and Future. Geneva, World Health Organization, 2017.

15 Frick M. Funding for TB Research Recent Momentum Must Inspire Bold Commitments. New York, Treatment Action Group, 2018.

16 Stop TB Partnership. The Global Plan to End TB, 2016-2020: the Paradigm Shift. Geneva, United Nations Office for Project Services/Stop TB Partnership, 2015. 